\title{
Semiclassical analysis of high harmonic generation in bulk crystals
}

\author{
G. Vampa, ${ }^{1, *}$ C. R. McDonald, ${ }^{1, \dagger}$ G. Orlando, ${ }^{1}$ P. B. Corkum, ${ }^{1,2}$ and T. Brabec ${ }^{1}$ \\ ${ }^{1}$ Department of Physics, University of Ottawa, Ottawa, ON K1N 6N5, Canada \\ ${ }^{2}$ National Research Council of Canada, Ottawa, ON, K1A OR6, Canada
}

(Received 7 October 2014; revised manuscript received 19 December 2014; published 23 February 2015)

\begin{abstract}
High harmonic generation (HHG) in solids is investigated. We find that interband emission is dominant for the midinfrared laser driver frequencies, whereas intraband emission dominates the far-infrared range. Interband HHG is similar to atomic HHG and therewith opens the possibility to apply atomic attosecond technology to the condensed matter phase. Interband emission is investigated with a quasiclassical method, by which HHG can be modeled based on the classical trajectory analysis of electron-hole pairs. This analysis yields a simple approximate cutoff law for HHG in solids. Differences between HHG in atoms and solids are identified that are important for adapting atomic attosecond technology to make it applicable to condensed matter.
\end{abstract}

DOI: 10.1103/PhysRevB.91.064302

PACS number(s): 42.65.Ky, 42.50.Hz, 78.47.-p, 72.20.Ht

\section{INTRODUCTION}

The interaction of intense laser fields with solid matter has been studied since the advent of nonlinear optics. Most of it happens in the perturbative realm, and the major outcome of such interactions is the parametric conversion of a few pump laser photons to a single photon of higher energy, i.e., a radiation field with frequency being a harmonic of the laser frequency is created [1]. The process of harmonic generation has been revolutionized by the availability of more intense lasers capable of converting tens to hundreds of photons to produce coherent soft $\mathrm{x}$-ray radiation. This phenomenon, dubbed high-order harmonic generation (HHG) [2,3], operates in a highly nonperturbative regime that cannot be understood with conventional nonlinear optics techniques. So far, research has focused on HHG in atomic and molecular gases. Only recently, high harmonics have been measured from the bulk of semiconductor crystals thereby opening the way to the study of nonperturbative optical phenomena in the condensed matter phase [4-6]. Experiments have been performed in two different wavelength domains, for mid-infrared (IR) [4] and for far-IR [terahertz (THz)] driving wavelengths [5,6]. Further, HHG has been studied via single- and two-color experiments. HHG occurs when electron-hole pairs are generated and subsequently accelerated by the same intense pump field [4,6]. In two-color experiments [5], conducted on semiconductor heterostructures, bound excitons are created by resonant excitation with a weak near-infrared field, followed by high harmonic sideband generation driven by $\mathrm{THz}$ fields. Our analysis focuses on single-color HHG in semiconductor bulk materials.

The physical mechanisms driving HHG in atoms and solids are closely related. In the limit of intense fields nonperturbative mechanisms dominate the perturbative nonlinear response. In atoms there are two main nonperturbative sources of harmonic emission: (i) when an electron is tunnel ionized and subsequently undergoes sinusoidal motion in the laser field, the nonlinear dependence of tunnel ionization on the laser field strength creates a nonlinear refractive index contributing to harmonic generation [7] and (ii) the oscillating free electron

\footnotetext{
*gvamp015@uottawa.ca

†cmcdo059@uottawa.ca
}

recollides with the atom, recombines to the ground state, and emits a high-energy photon [2]. Whereas the first mechanism contributes mainly to low-order harmonic generation, recollision dominates generation of the higher harmonic orders.

HHG in solids behaves very similarly [8,9], with electrons and holes moving in the conduction and valence bands, respectively. The ionization step corresponds to a transition of electrons from the valence (lower) to the conduction (higher) band. The first mechanism is richer in solids than in atoms, due to a different relation between energy and momentum: the band structure contains anharmonic terms beyond second order in the crystal momentum. These terms result in a nonlinear dependence of the band velocity and therewith of the current on the laser field resulting in HHG [10]. As a result, both, tunnel ionization and the nonlinear band velocity, contribute to harmonic generation. These contributions are termed intraband HHG, as they arise from the nonsinusoidal dynamics of electrons and holes in their respective conduction and valence bands. The second (interband) mechanism is due to the build-up of polarization between the valence and conduction band [6,11], and is analogous to recollision in atoms [9]. In theoretical investigations for $\mathrm{THz}$ drivers it was suggested that an interplay between intra- and interband emission is responsible for $\mathrm{HHG}$ [6]. By contrast, so far, intraband $\mathrm{HHG}$ has been assumed to be responsible for high harmonic emission [12-14] with mid-IR driver frequencies. A recent theoretical investigation comparing inter- and intraband $\mathrm{HHG}$ for mid-IR driving laser wavelengths [9] found that interband $\mathrm{HHG}$ is the dominant contribution. A strong similarity of atomic HHG and mid-IR driven interband HHG in solids was demonstrated.

Atomic HHG is the foundation on which attosecond science is built. It is fundamental to the realization of attosecond XUV pulses that allow for electron dynamics to be studied with unprecedented temporal and spatial resolution in pump-probe experiments [15-18]. Furthermore, it allows for the generating medium $[19,20]$ and its interaction with strong fields to be probed [21-23]. The similarity of atomic HHG and mid-IR driven interband HHG in solids [9] opens the potential to adapt and apply the rich reservoir of atomic attosecond technology to the condensed matter phase.

In this work, we build on Ref. [9] to gain a deeper understanding of harmonic generation in solids, a prerequisite 
for adapting atomic attosecond methodology to condensed matter systems. We begin by exploring interband versus intraband emission in more detail and over a broader wavelength range. This reveals that recollision and interband $\mathrm{HHG}$ dominates only for mid-IR driving laser wavelengths. As dephasing increasingly suppresses recollision and interband $\mathrm{HHG}$, intraband emission becomes the favored mechanism for driving lasers in the far-IR regime.

The rest of the paper treats interband HHG in the mid-IR regime. Similar to atomic $\mathrm{HHG}$, quasiclassical equations are derived by applying saddle point integration to the interband current cast into an integral form by using the Keldysh approximation $[9,24]$. The main focus of this work lies on a detailed analysis of the quasiclassical trajectories obtained from these equations. Based on the trajectories, a number of important properties of interband HHG can be explained.

(i) In the absence of dephasing, the harmonic spectrum shows a continuum like structure due to interference from multiple collisions. Only for dephasing times comparable to a laser half cycle, a clean harmonic spectrum emerges. The presence of dephasing, in the form of collisions, destroys the coherence between the electron and the hole. As a result, harmonic emission from higher re-encounters occurring at times greater than the dephasing time are suppressed.

(ii) A cutoff law is derived and its prediction agrees with numerical simulations. The maximum obtainable harmonic energy in the two-band approximation is capped by the maximum energy difference between valence and conduction bands [11].

(iii) Differences between HHG in atoms and solids are analyzed. We find that at low field strengths the trajectories are similar to those in atoms. By contrast, for higher field strengths, the trajectories are strongly modified. As a consequence of the anharmonic band structure and the boundedness of the cutoff, those trajectories that would create harmonic energies above the maximum band gap appear at lower energies with modified recombination/emission times. This has profound implications for the application of atomic attosecond technologies, such as the synthesis of subfemtosecond pulses, to the condensed matter phase.

The article is organized as follows. In the first section, we review the theoretical framework. In Sec. III, the importance of interband and intraband HHG is investigated as a function of the driving laser wavelength. In this section, the band structure of $\mathrm{ZnO}$ is used, as obtained from numerical analysis [25]. In the remaining sections the $\mathrm{ZnO}$ band structure is simplified by using a nearest neighbor (NN) approximation. In Sec. IV, we derive an intuitive recollision model for interband harmonic emission, and in Secs. V and VI, we use it to extract an approximate cutoff law and study the electron-hole trajectories in more detail.

\section{THEORETICAL MODEL}

Our analysis of the interaction of strong lasers with $\mathrm{ZnO}$ crystals is based on a $3 \mathrm{D}$ two-band single active electron model solid with general band dispersion,

$$
E_{m}(\mathbf{k})=E_{m, x}\left(k_{x}\right)+E_{m, y}\left(k_{y}\right)+E_{m, z}\left(k_{z}\right)
$$

TABLE I. Coefficients of the expansion of the NL-EPM bands from Ref. [25] for the crystal-field split valence band and the first conduction band.

\begin{tabular}{lrr}
\hline \hline & Valence band & Conduction band \\
\hline$a_{0, x}$ & -0.0928 & 0.0898 \\
$a_{1, x}$ & 0.0705 & -0.0814 \\
$a_{2, x}$ & 0.0200 & -0.0024 \\
$a_{3, x}$ & -0.0012 & -0.0048 \\
$a_{4, x}$ & 0.0029 & -0.0003 \\
$a_{5, x}$ & 0.0006 & -0.0009 \\
$a_{0, y}$ & -0.0307 & 0.1147 \\
$a_{1, y}$ & 0.0307 & -0.1147 \\
$a_{0, z}$ & -0.0059 & 0.0435 \\
$a_{1, z}$ & 0.0059 & -0.0435 \\
\hline \hline
\end{tabular}

with $m=(v, c)$ for the valence and conduction bands, respectively. We use the lowest conduction band and the crystal field split valence band in our calculations [25]. For each direction in the reciprocal space $(i=x, y, z)$,

$$
\begin{gathered}
E_{\mathrm{v}, i}\left(k_{i}\right)=\sum_{j=0}^{\infty} \alpha_{\mathrm{v}, i}^{j} \cos \left(j k_{i} a_{i}\right), \\
E_{\mathrm{c}, i}\left(k_{i}\right)=E_{g}+\sum_{j=0}^{\infty} \alpha_{\mathrm{c}, i}^{j} \cos \left(j k_{i} a_{i}\right)
\end{gathered}
$$

with $a_{i}=2 \pi / \kappa_{i}$ where $\kappa_{i}$ the reciprocal space wave vector along coordinate $i$. The orientation of the reciprocal lattice of $\mathrm{ZnO}$ (wurtzite structure) is chosen so that $\hat{\mathbf{x}}\|\Gamma-M, \hat{\mathbf{y}}\|$ $\Gamma-K$, and $\hat{\mathbf{z}} \| \Gamma-A$ (optical axis); along these directions [25] $\left(a_{x}, a_{y}, a_{z}\right)=(5.32,6.14,9.83)$ a.u. Atomic units are used throughout the paper unless otherwise indicated. The expansion coefficients $\alpha_{\mathrm{m}, i}^{j}(i=x, y, z, j=0,1 \ldots)$ for valence $(\mathrm{m}=\mathrm{v})$ and conduction $(\mathrm{m}=\mathrm{c})$ band are obtained from expanding the bands calculated with a nonlocal empirical pseudopotential method (NL-EPM, described in Ref. [25]) up to $j=5$ for $\Gamma-M$; the nearest-neighbor expansion is used for $\Gamma-K$ and $\Gamma-A$. The resulting coefficients are listed in Table I. These bands are plotted in Fig. 1. The band-gap energy at the $\Gamma$ point is given by $E_{\mathrm{g}}=0.1213$ a.u. $(3.3 \mathrm{eV})$. It should be noted here that, in Ref. [9], both the local density approximation (LDA-ABINIT) and NL-EPM were used to determine the $\mathrm{ZnO}$ band structure. However, only the spectra using the LDA-ABINIT bands were shown. In this work we will show the spectra obtained using the NL-EPM bands (for $\Gamma-M$ ). While some quantitative differences exist, the conclusions remain unchanged. This highlights the difficulty in obtaining highly-accurate band structures for $\mathrm{ZnO}$.

There exist two main mechanisms/sources for the generation of harmonic radiation in solids. Under the action of the strong laser field, electrons tunnel to the conduction band, leaving holes in the valence band. The electron-hole pairs thus formed are subsequently accelerated by the field. High harmonics are generated as a result of the electron's and hole's independent motion in their respective bands (intraband current) and of the polarization buildup between 


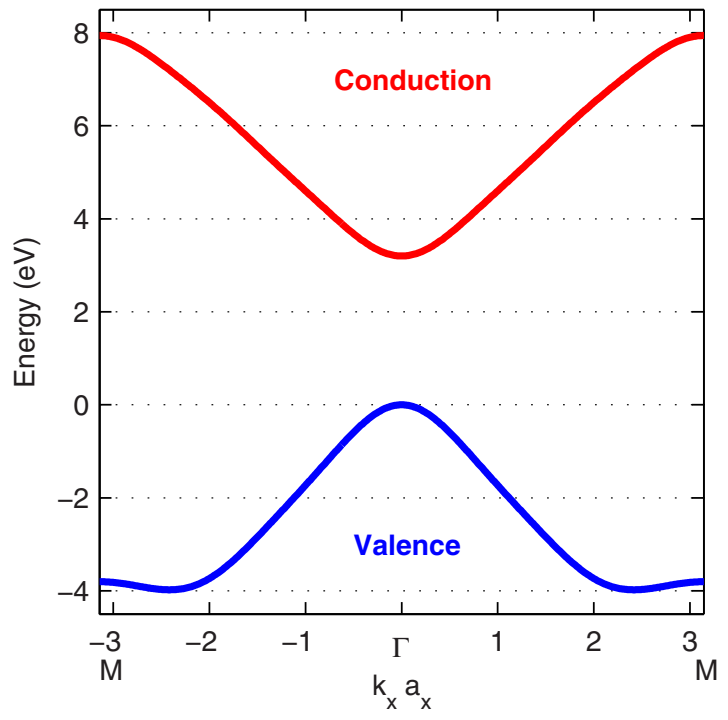

FIG. 1. (Color online) The first conduction (red line) and the split-off valence (blue line) bands along the $\Gamma-M$ direction of the $\mathrm{BZ}$ are extracted from Ref. [25]; for the expansion coefficients see Table I.

them (interband current). These currents can be calculated as [9]

$$
\begin{aligned}
& \mathbf{j}_{\mathrm{ra}}(t)=\sum_{\mathrm{m}=\mathrm{c}, \mathrm{v}} \int_{\mathrm{BZ}} \mathbf{v}_{\mathrm{m}}(\mathbf{K}+\mathbf{A}(t)) n_{m}(\mathbf{K}, t) d^{3} \mathbf{K}, \\
& \mathbf{j}_{\mathrm{er}}(t)=\frac{d}{d t} \int_{\mathrm{BZ}} \mathbf{p}(\mathbf{K}, t) d^{3} \mathbf{K},
\end{aligned}
$$

where the band velocity is defined by $\mathbf{v}_{\mathrm{m}}(\mathbf{k})=\nabla_{\mathbf{k}} E_{\mathrm{m}}(\mathbf{k})$, $\mathbf{A}(t)=-\mathbf{A}_{\mathbf{0}} \sin (\omega t)$ is the laser vector potential, $\mathbf{F}(t)=$ $-d \mathbf{A} / d t=\mathbf{F}_{\mathbf{0}} \cos (\omega t)$ is the laser electric field, and crystal momentum $\mathbf{k}$ has been transformed into a frame moving with the vector potential, $\mathbf{K}=\mathbf{k}-\mathbf{A}(t)$. In experiments [4], the vacuum field strength $F_{\mathrm{v}}$ is measured. Due to surface reflection (here considered at normal incidence) the peak electric field strength in the material is reduced to $F_{0}=2 F_{\mathrm{v}} /(n+1)$, with $n=1.8992$ the material refractive index at the fundamental frequency. The high harmonic spectrum is obtained from the Fourier transform (FT) of $\mathbf{j}_{\mathrm{t}}=\mathbf{j}_{\mathrm{ra}}+\mathbf{j}_{\mathrm{er}}$, as $\left|\mathrm{FT}\left\{\mathbf{j}_{\mathrm{t}}\right\}\right|^{2}$. The population of band $\mathrm{m}, n_{\mathrm{m}}(\mathbf{K}, t)$, and the interband polarization $\mathbf{p}(\mathbf{K}, t)$ are governed by the semiconductor Bloch equations:

$$
\begin{gathered}
\dot{\pi}(\mathbf{K}, t)=-\frac{\pi(\mathbf{K}, t)}{T_{2}}-i \Omega(\mathbf{K}, t) w(\mathbf{K}, t) e^{-i S(\mathbf{K}, t)}, \\
\dot{n}_{\mathrm{m}}(\mathbf{K}, t)=i s_{m} \Omega^{*}(\mathbf{K}, t) \pi(\mathbf{K}, t) e^{i S(\mathbf{K}, t)}+\text { c.c. },
\end{gathered}
$$

Here, $w=n_{\mathrm{v}}-n_{\mathrm{c}}$ is the population difference, $S(\mathbf{K}, t)=$ $\int_{-\infty}^{t} \varepsilon_{\mathrm{g}}\left(\mathbf{K}+\mathbf{A}\left(t^{\prime}\right)\right) d t^{\prime}$ is the classical action, $\varepsilon_{\mathrm{g}}=E_{\mathrm{c}}-E_{\mathrm{v}}$ is the band gap between conduction and valence bands, $\Omega(\mathbf{K}, t)=\mathbf{F}(t) \mathbf{d}(\mathbf{K}+\mathbf{A}(t))$ is the Rabi frequency, and $s_{m}=$ $-1,1$ for $\mathrm{m}=\mathrm{v}, \mathrm{c}$, respectively.

Finally, $\pi(\mathbf{K}, t)$ is related to the polarization $\mathbf{p}(\mathbf{K}, t)$ by

$$
\mathbf{p}(\mathbf{K}, t)=\mathbf{d}(\mathbf{K}+\mathbf{A}(t)) \pi(\mathbf{K}, t) e^{i S(\mathbf{K}, t)}+\text { c.c. },
$$

where $\mathbf{d}(\mathbf{k})=i \int d^{3} \mathbf{x} u_{\mathrm{v}, \mathbf{k}}^{*}(\mathbf{x}) \nabla_{\mathbf{k}} u_{\mathrm{c}, \mathbf{k}}(\mathbf{x})$ is the transition dipole moment, with $u_{\mathrm{m}, \mathbf{k}}$ the periodic part of the Bloch function; $\mathbf{d}=(3.46,3.46,3.94)$ is assumed to be $k$-independent in our analysis [9]. Initially, all electrons are in the valence band. For a more detailed derivation see Ref. [9].

Equation (4) describe the creation and annihilation of electron-hole pairs and their dynamics in their respective bands. The acceleration of the electron-hole pairs by the laser field, and therefore the relative transfer of population between different crystal momenta $\mathbf{k}$, occurs through the temporal variation of $\varepsilon_{g}\left(\mathbf{K}+\mathbf{A}\left(t^{\prime}\right)\right)$ in the action $S(\mathbf{K}, t)$. This mechanism is the main driver of the HHG process, as is confirmed by the semiclassical analysis of Sec. IV.

Multielectron effects are neglected in our treatment. We expect that the excitonic electron-hole interaction will result in corrections, but will not substantially alter the high harmonic generation mechanism-similar to what was found for atomic HHG. Other many-body effects, such as band-gap renormalization, will introduce new features that were not observed in atomic HHG, as they are characteristic of the condensed matter phase. Dephasing of the interband polarization is introduced in Eq. (4) in the relaxation-time approximation, through the parameter $T_{2}$. It is a parameter of our simulations and its effects on the high harmonic spectrum are investigated in the next section.

\section{DISCUSSION OF HIGH HARMONIC SPECTRA}

Figure 2 shows the high harmonic spectrum of the intraband (red) and interband (blue) currents obtained from a wurtzite $\mathrm{ZnO}$ crystal for two dephasing times $T_{2}$ for field strength $F_{0}=0.003$ a.u. $(0.15 \mathrm{~V} / \AA)$, (mid-IR) laser central frequency $\omega=0.014$ a.u. $(3.25 \mu \mathrm{m})$, and laser polarization and wave

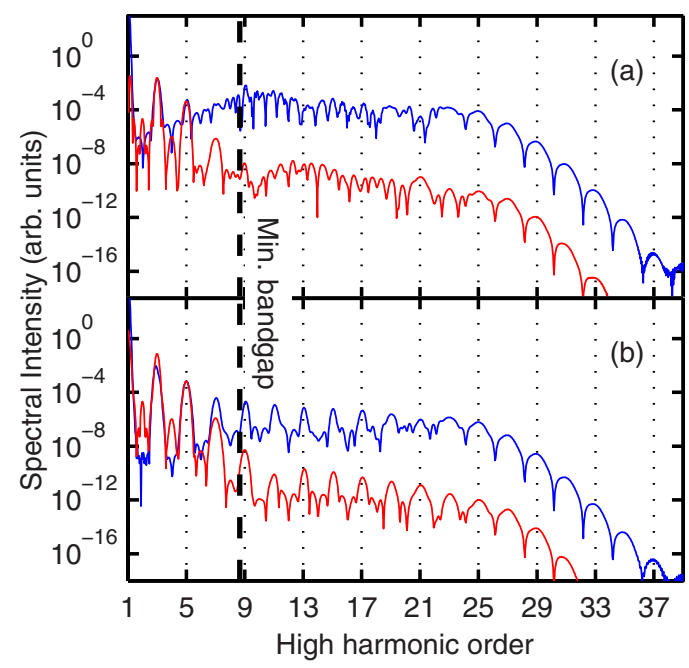

FIG. 2. (Color online) Harmonic spectrum for interband $\left(\left|F T\left\{\mathbf{j}_{\text {er }}\right\}\right|^{2}\right.$, blue line) and intraband $\left(\left|F T\left\{\mathbf{j}_{\text {ra }}\right\}\right|^{2}\right.$, red line) currents for a field strength $F_{0}=0.003$ a.u.; we use a temporal Gaussian envelope with a FWHM of ten cycles and cosine carrier with frequency $\omega=0.014$ a.u., corresponding to a laser period $T_{0}=2 \pi / \omega=10.9$ fs. The dephasing time is (a) $T_{2}=\infty$ and (b) $T_{2}=T_{0} / 4$. The laser polarization is parallel to the $\Gamma-M$ direction of the reciprocal space of a wurtzite $\mathrm{ZnO}$ crystal. The dashed black vertical line represents the minimum band gap at the center of the Brillouin zone. 
vector aligned along the $\Gamma-M$ and $\Gamma-A$ directions of the reciprocal space, respectively. The currents are calculated from Eq. (3) with $n_{m}(\mathbf{K}, t)$ and $\mathbf{p}(\mathbf{K}, t)$ obtained by a 3D numerical integration of Eq. (4). The same method is used as in Ref. [9].

The spectrum of both harmonic sources depends on the dephasing time. Figure 2(a) shows the harmonic spectrum in the absence of dephasing. Contrary to experimental findings, no clear harmonic structure is observed. Only for dephasing times equal to or shorter than a laser half cycle does the harmonic structure become resolved; see Fig. 2(b) for $T_{2}=$ 2.7 fs corresponding to a laser quarter cycle. This finding has been explained in Ref. [9] in the following way.

Interband HHG occurs through laser induced tunneling and creation of electron-hole pairs, which are accelerated back and forth in the laser field. When they re-encounter, the electron hole pair recombines and a harmonic photon is created. The structure of the interband harmonics is washed out by interference between the first and higher order recollisions, see Fig. 2(a). Only when higher-order recollisions are suppressed, $T_{2} \leqslant T_{0} / 2$, the odd harmonic spectrum becomes clearly visible. Even for interband harmonics below the band gap, recollision appears to remain the dominant mechanism, as the odd harmonic structure can only be observed for $T_{2} \leqslant T_{0} / 2$. Note that for harmonics above the cutoff only a single recollision is possible, which is why the odd harmonic structure remains visible even in the absence of dephasing.

Intraband HHG occurs through three main mechanisms: it can by driven by the nonlinearity of the band velocities, by the nonlinearity arising from tunnel ionization [7,14], or it can result from scattering during recollision of electron and hole [9]. For intraband harmonics above the band gap, the recollision contribution dominates the other two, as the intraband spectrum in Fig. 2 is blurred in the absence of dephasing, similar to interband HHG. Below the band gap, a clear intraband $\mathrm{HH}$ spectrum can be seen in the absence of dephasing. This indicates that for below band gap intraband harmonics, the other two nonrecollision mechanisms are dominant.

Finally, above the band gap, interband HHG is dominant, which was confirmed for a wide range of laser intensities $\left(F_{0}=\right.$ $0.003-0.1)$. For harmonics below the band gap in Fig. 2, intraband emission is dominant instead. However, no universal conclusion can be drawn, as the relative strength of inter- and intraband HHG below the band gap is found to be sensitive to laser intensity. For example, increasing the field strength to $F_{0}=0.0058$ and leaving all other parameters in Fig. 2 unchanged, we find interband HHG to be dominant for most of the harmonics below the band gap.

So far, we have investigated inter- and intraband HHG for one mid-IR laser wavelength. However, HHG experiments in solids have also been performed in the far-IR (THz) range $[5,6]$. Therefore it is interesting to compare the relative importance of the two emission mechanisms over a broader range of laser wavelengths. Figure 3 compares the spectral intensity of interband (solid lines) and intraband (dashed lines) HHG for several harmonic orders as a function of laser wavelength. All other parameters are the same as in Fig. 2. The arrows mark the wavelengths above which a given harmonic order falls below the minimum band gap. As long as HHG is above the band gap, interband emission dominates.

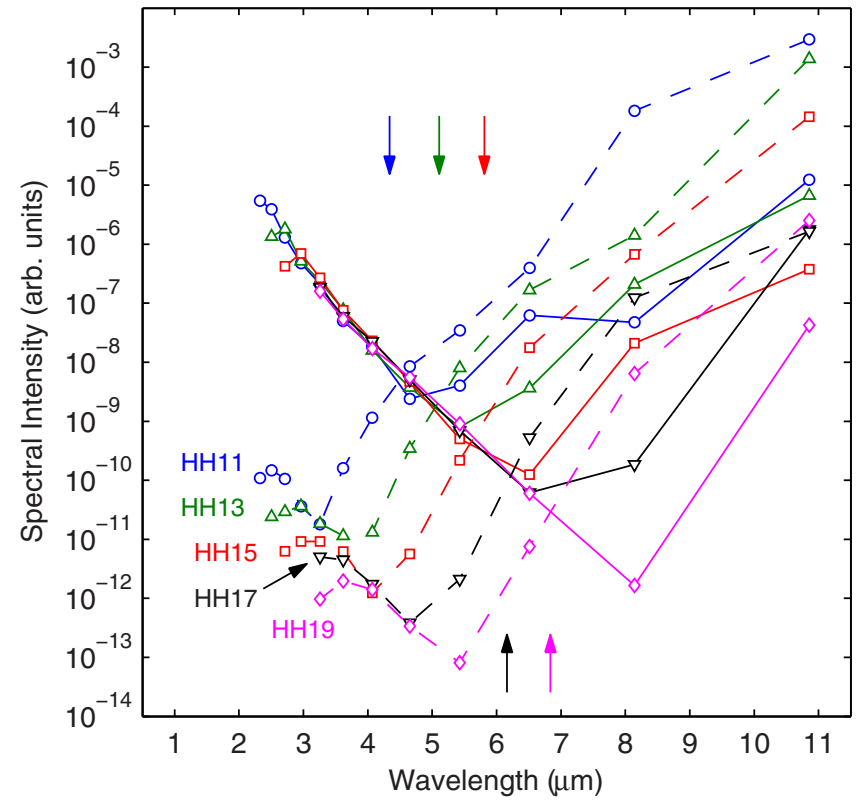

FIG. 3. (Color online) Harmonic intensity vs laser wavelength for various high harmonic $(\mathrm{HH})$ orders; laser and band parameters are as in Fig. 2; circles, triangles pointed upward, squares, triangles pointed downward, and diamonds refer to $\mathrm{HH}$ order 11, 13, 15, 17, and 19, respectively. The solid and dashed lines show the interband and intraband contributions for $T_{2}=2.7 \mathrm{fs}\left(T_{0} / 4\right.$ for $\left.\lambda=3.25 \mu \mathrm{m}\right)$, respectively. The arrows mark the wavelength above which a given harmonic falls below the minimum band gap.

Around the position of the arrows, where the harmonics slip below the band gap, intraband HHG becomes dominant. The exact point at which inter- and intraband yield cross is laser intensity dependent. Over the range of investigated field strengths $\left(F_{0}=0.003-0.1\right)$, the crossing wavelength increases with increasing field strength and varies over a range of about $1 \mu \mathrm{m}$. Thus our analysis indicates that the intraband mechanism dominates HHG in solids in the far-IR laser wavelength regime. In particular, nonrecollision mechanisms are expected to be dominant in this range, as the ratio $T_{2} / T_{0}$ is reduced and the relative importance of dephasing is augmented for increasing wavelength, suppressing more and more the recollision contribution to HHG.

In the rest of the paper, we will focus on the mid-IR laser wavelength range and on above band gap harmonics for which interband HHG is dominant. Harmonic generation in this regime is very promising, as the mechanism of interband emission is closely related to HHG in atoms. This presents the opportunity to adapt attosecond metrology methods developed for atoms and apply them to solids. Our analysis will focus on the saddle point integration of interband HHG and on the resulting quasiclassical trajectory formalism. The intuitive picture revealed by the trajectory analysis presents a useful basis for developing attosecond methodology for the condensed matter phase.

\section{DERIVATION OF TRAJECTORIES}

In order to better understand the physical process driving interband HHG in solids, it is useful to consider the limit of 
low ionization where $n_{v}-n_{c} \approx 1$. In this limit, the interband spectrum can be cast into the following integral form [9]:

$$
\begin{aligned}
\mathbf{j}_{\mathrm{er}}(\Omega)= & \Omega \int_{\mathrm{BZ}} d^{3} \mathbf{k} \mathbf{d}(\mathbf{k}) \int_{-\infty}^{\infty} d t e^{i \Omega t} \int_{-\infty}^{t} d t^{\prime} F\left(t^{\prime}\right) d^{*}\left(\boldsymbol{\kappa}_{t^{\prime}}\right) \\
& \times e^{-i S\left(\mathbf{k}, t^{\prime}, t\right)-\left(t-t^{\prime}\right) / T_{2}}+\text { c.c. },
\end{aligned}
$$

where $\boldsymbol{\kappa}_{t^{\prime}}=\mathbf{k}+\mathbf{A}\left(t^{\prime}\right)-\mathbf{A}(t)$ and $S\left(\mathbf{k}, t^{\prime}, t\right)=\int_{t^{\prime}}^{t} \varepsilon_{\mathrm{g}}\left(\boldsymbol{\kappa}_{\tau}\right) d \tau$ is the action.

Similar to atomic HHG [26], the integrals can be evaluated with the saddle point method. The dominant contributions to the integral occur at the stationary phase points, for which the first derivative of the phase $S$ is zero. The resulting saddle point conditions are

$$
\begin{aligned}
\nabla_{\mathbf{k}} S & =\int_{t^{\prime}}^{t} \mathbf{v}\left(\mathbf{k}-\mathbf{A}(t)+\mathbf{A}\left(t^{\prime \prime}\right)\right) d t^{\prime \prime}=0, \\
\frac{d S}{d t^{\prime}} & =\varepsilon_{\mathrm{g}}\left(\mathbf{k}-\mathbf{A}(t)+\mathbf{A}\left(t^{\prime}\right)\right)-\frac{i}{T_{2}}=0, \\
\frac{d S}{d t} & =\varepsilon_{\mathrm{g}}(\mathbf{k})-\Omega+\frac{i}{T_{2}}=0,
\end{aligned}
$$

where $\mathbf{v}(\mathbf{k})=\nabla_{\mathbf{k}} \varepsilon_{\mathrm{g}}(\mathbf{k})=\mathbf{v}_{\mathrm{c}}-\mathbf{v}_{\mathrm{v}}$ is the difference of the velocities of the electron and the hole in their respective bands. Further, $\mathbf{x}_{\mathrm{c}, \mathrm{v}}\left(t^{\prime}, t\right)=\int_{t^{\prime}}^{t} d t^{\prime \prime} \mathbf{v}_{\mathrm{c}, \mathrm{v}}$ is the space excursion of electron and hole.

The resulting saddle points in $\mathbf{k}, t, t^{\prime}$ are functions of the harmonic frequency $\Omega$ and are in general complex. The imaginary parts arise from the tunneling of the electron from the valence to the conduction band, which is a purely quantum-mechanical process [26,27]. The six equations for real and imaginary parts of the three saddle points are coupled and have to be solved self consistently. However, to first order, the equations for the imaginary parts can be neglected, which gives a good approximation of the classical electron and hole trajectories after their birth through tunnel ionization. In this case, the saddle point conditions have a simple physical interpretation.

Equation (7a), $\nabla_{\mathbf{k}} S=\Delta x_{c}-\Delta x_{v}=0$, states that the total distance traveled by the electron equals that traveled by the hole. Since they are born at the same position, the condition implies that high harmonics are emitted only upon re-encounter of electron and hole.

In the limit $T_{2}=\infty$, Eqs. (7b) and (7c) also have a classical interpretation. The first one defines the birth time at which the electron-hole pair is formed by tunnel ionization. It also determines the quasiclassical evolution of electron and hole crystal momentum in the laser field. The second condition ensures conservation of energy: the electron recombines with the hole and emits one photon with energy equal to the energy band gap at the crystal momentum of recombination.

Thus the essence of high harmonic generation from solids is similar to the three-step model of atomic HHG: an electronhole pair is created at time of birth $t^{\prime}$ (step one); the electron and hole are accelerated by the field in opposite directions (step two); when the field changes sign they are driven back towards their point of origin and eventually re-encounter each other at time $t$ with nonzero crystal momentum. The crystal momentum determines the band gap energy difference between electron and hole and therewith the energy of the photon that is emitted upon recombination.

Because the minimum band gap is never zero, Eq. (7b) implies a complex birth time. In fact, by writing $\varepsilon_{g}(\mathbf{k})=E_{g}+$ $h(\mathbf{k})$, where $E_{g}$ is the minimum band gap of the material and $h(\mathbf{k}) \geqslant 0$, Eq. (7b) reads

$$
h\left(\mathbf{k}-\mathbf{A}(t)+\mathbf{A}\left(t^{\prime}\right)\right)=-E_{g},
$$

which can only be solved for a complex $t^{\prime}$. The classical approximation implies setting $E_{g}=0$, which allows for a real solution. The tunneling process and its effects on the electron-hole pair trajectories are then disregarded. For direct band-gap materials, for which the minimum band gap is at the $\Gamma$ point $(k=0)$, the solution of the resulting equation is

$$
\mathbf{k}=\mathbf{A}(t)-\mathbf{A}\left(t^{\prime}\right)
$$

which is the classical equation of motion for electron-hole pairs in a lattice (see, for example, Ref. [10], Chap. 12). Regarded as a function of $t$ the above equation implies that the pairs are born with zero momentum at time $t^{\prime}$ (at the $\Gamma$ point). In indirect band-gap materials, where the minimum band gap is not at the $\Gamma$ point, electron-hole pairs are created with (possibly large) nonzero crystal momentum. In our view, this represents an interesting point for further study, theoretically as well as experimentally.

We solve the saddle point equations in the classical approximation as follows. For each birth time $t^{\prime}$, an electron and a hole are propagated with velocity $\mathbf{v}[\mathbf{k}(\tau)]=\mathbf{v}\left[\mathbf{A}(\tau)-\mathbf{A}\left(t^{\prime}\right)\right]$ until time $t$ when they re-encounter with momentum $\mathbf{k}=\mathbf{k}(t)$. Equations (7a) and (7b) are thus satisfied. The two stationary times define a classical trajectory, which is associated to a high harmonic photon energy $\varepsilon_{g}(\mathbf{k})$ via Eq. (7c). As a result, in a two band system the highest achievable harmonics are determined by the maximum band gap between valence and conduction bands, in agreement with what was found in previous theoretical studies [11].

The classical description is used in the following two sections to derive an approximate high harmonic cutoff law and to discuss the physics driving interband HHG based on a trajectory analysis. The main difference between HHG in atoms and solids comes from the fact that most of the band structure in nonparabolic. Electrons and holes can be approximated as free particles only in the vicinity of the $\Gamma$ point, where the bands are a quadratic function of the crystal momentum. This holds only for harmonic orders close to the minimum band-gap energy. In this range, HHG in atoms and solids are very similar. For higher harmonics, electrons and holes explore the nonparabolic parts of the band structure, displaying different effects from those normally present in atomic HHG.

\section{DERIVATION OF THE CUTOFF LAW}

The saddle point analysis of the interband emission mechanism is particularly useful in that it allows to predict the cutoff photon energy. Conservation of energy, Eq. (7c), requires that the cutoff harmonic $\Omega_{c}$ is determined by electron-hole pairs recolliding with the highest momentum $\mathbf{k}_{c}$, that is,

$$
\Omega_{c}=\varepsilon_{\mathrm{g}}\left(\mathbf{k}_{c}\right) .
$$


As a result, the variation of the cutoff with field strength directly reflects the momentum dependent band gap: as the crystal momentum increases linearly with field strength, a band gap that is a linear function of the crystal momentum yields a linear dependence of the cutoff harmonic on the driving radiation field. Likewise, a parabolic dispersion yields a cutoff that scales quadratically with the field, or linearly with the intensity. This is the case in atomic HHG, where the energy of the electron in vacuum varies quadratically with momentum.

In a solid, the band energy can be a complicated function of momentum. Fortunately, in most cases, $\varepsilon_{g}(\mathbf{k})$ is almost linear near the half-point of the BZ-between $\Gamma$ and $\kappa_{i} / 2$-as the curvature changes sign between the bottom and the top of the bands. We use this property to derive an approximate analytical scaling law of the cutoff as a function of laser and crystal parameters. By expanding $\varepsilon_{g}(\mathbf{k})$ up to first order around $k=\kappa_{x} / 4$, we obtain

$$
\Omega_{c} \simeq \varepsilon_{g}\left(\frac{\kappa_{x}}{4}\right)+\left.\frac{d \varepsilon_{g}\left(k_{c}\right)}{d k_{c}}\right|_{k_{c}=\kappa_{x} / 4}\left(k_{c}-\frac{\kappa_{x}}{4}\right) .
$$

The momentum of recollision is determined by a trajectory born at time $t_{c}^{\prime}$ and recolliding at time $t_{c}$,

$$
k_{c}=\frac{F_{0}}{\omega}\left[\sin \left(\omega t_{c}^{\prime}\right)-\sin \left(\omega t_{c}\right)\right]=\frac{F_{0} \delta}{\omega} .
$$

This trajectory can be found with the numerical procedure described in the previous section. In the following, we will discuss the case of nearest neighbours (NN) bands, where $a_{0 c}=\Delta_{c}, a_{1 c}=-\Delta_{c}, a_{0 v}=-\Delta_{v}, a_{1 v}=\Delta_{v}$. Inserting the $\mathrm{NN}$ approximation in Eq. (11) yields the cutoff law

$$
\Omega_{c}=E_{\mathrm{g}}+\left(\Delta_{\mathrm{c}}+\Delta_{\mathrm{v}}\right)\left(1-\frac{\pi}{2}+\frac{F_{0} a_{x} \delta}{\omega}\right) .
$$

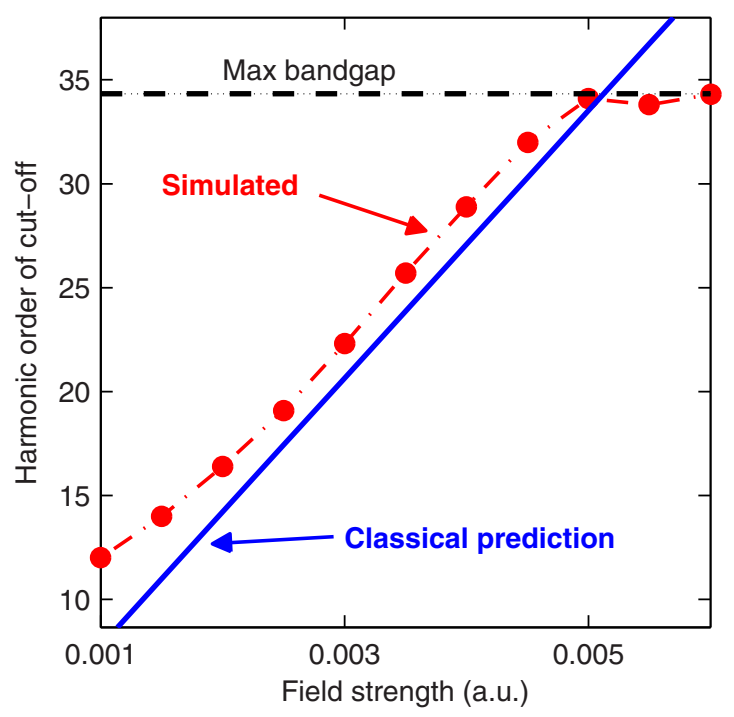

FIG. 4. (Color online) The simulated high harmonic cutoff (red circles, red dash-dotted line) lies slightly above the cutoff predicted with classical trajectory analysis [Eq. (13)]. The band parameters are given in the caption of Table II. The laser polarization is aligned along the $\Gamma-M$ direction of the hexagonal Brillouin zone of $\mathrm{ZnO}$, the central frequency is $\omega=0.014$ a.u.
TABLE II. Parameters describing the cut-off trajectory as a function of field strength for a NN band structure with coefficients: $\Delta_{\mathrm{c}}=0.0872$ a.u., $\Delta_{\mathrm{v}}=0.0925$ a.u., a $=10.64$ a.u., and $E_{\mathrm{g}}=$ 0.1213 a.u. Birth $\left(t^{\prime}\right)$ and recollision $(t)$ times are given in units of optical cycles $\left(T_{0}\right)$. The trajectory responsible for the cutoff emission changes slightly as a function of the laser field strength.

\begin{tabular}{lcccc}
\hline \hline$F_{0}$ & $t^{\prime} / T_{0}$ & $t / T_{0}$ & $\delta$ & Two-band cutoff \\
\hline 0.001 & 0.050 & 0.701 & 1.26 & 10.1 \\
0.002 & 0.052 & 0.702 & 1.27 & 14.3 \\
0.003 & 0.057 & 0.700 & 1.30 & 20.4 \\
0.004 & 0.063 & 0.708 & 1.35 & 27.5 \\
0.005 & 0.077 & 0.728 & 1.45 & 33.4 \\
0.006 & 0.107 & 0.630 & 1.35 & 34.3 \\
\hline \hline
\end{tabular}

The blue line in Fig. 4 represents the cutoff calculated with the above formula for a NN band structure that best approximates the real $\mathrm{ZnO}$ bands used for Fig. 2. The band parameters are reported in the caption of Table II. Table II reports the birth and recollision times for the cutoff trajectory along with $\delta$ defined in Eq. (12) for increasing field strengths. As Eq. (13) is based on an expansion around the crystal momentum at half BZ, $\delta$ can be taken from the trajectory that recollides at $\Omega_{c}=\varepsilon_{g}\left[\kappa_{x} / 4\right]$. This yields $\delta \simeq 1.32$.

The validity of Eq. (13) is tested against a simulation of the high harmonic spectrum obtained as described in Sec. II. Instead of the full band model we have also used the $\mathrm{NN}$ band defined above in the numerical solution to make a comparison with Eq. (13) possible. The cutoff is extracted from a windowed Fourier transform of the interband current, where a 0.34 cycles wide Blackman window is scanned across one optical cycle around the peak of the laser pulse. The resulting spectrum is continuous, rather than composed of discrete harmonics, because only one recollision event is allowed inside the narrow temporal window. The cutoff corresponds to the maximum photon energy in the plateau region of the spectrum, before the exponential roll-off of the high harmonic intensity (as in Fig. 2). This photon energy is plotted in Fig. 4. As a result of the continuous spectrum, this method provides a more accurate way of determining the cutoff than by inferring it from the discrete harmonic structure. As Fig. 4 shows, the numerical result (red circles and red dashed line) lies about one to two harmonic orders above the theoretical prediction (blue line), but their slope is identical.

The small difference between numerical and analytical cutoff is probably due to the neglect of the imaginary parts of the saddle points. Taking into account the complex nature of the saddle points [26,27], electrons and holes are not born at the same position $\Delta \mathrm{x}\left(t^{\prime}\right)=\mathrm{x}_{c}\left(t^{\prime}\right)-\mathrm{x}_{v}\left(t^{\prime}\right) \neq 0$. For recollision to occur, the electron and the hole have to travel the extra distance $\Delta x\left(t^{\prime}\right)$. The work done by the field gives the pair an extra energy $\delta \Omega=F(t) \Delta \mathrm{x}\left(t^{\prime}\right)$. A classical description fails to account for this fact and returns a lower cutoff.

In our two-band model, the maximum attainable photon energy is limited by the maximum band gap (black dashed line), in agreement with our discussion above. Higher harmonics are possible but require excitation to higher bands. If the electron reaches the top of the first conduction band 
it can tunnel to the second one and gain additional energy. This effect has been investigated in Ref. [28]. At the laser frequency considered in this work and for polarization aligned along the $\Gamma-M$ direction, electrons start to reach the edge of the BZ for $F_{0} \geqslant 0.0045$ a.u. However, the band gap between the two conduction bands is too large to result in substantial population transfer for the field strengths considered here. The role of recollision in higher bands is an extremely interesting venue of research that will be pursued in future work.

The derived cutoff law is accurate as long as the band gap is approximately a linear function of momentum. Deviations from the cutoff law are expected around the minimum and maximum energies, where quadratic terms dominate the dispersion. Indeed, deviations from the linear scaling are evident in Fig. 4 near the lowest and the highest photon energies. Most notably, Eq. (13) fails in the limit $F_{0} \rightarrow 0$ since it predicts a cutoff lower than the minimum band gap. In our quasiclassical analysis, the energy available to the electron-hole pair for recombination must always be higher or equal to $E_{g}$.

The classical analysis can also be used to estimate the validity of the one electron-hole exciton approximation used in our work. Whether coherent emission can arise from an electron recolliding with a different hole than the one that it created upon ionization, or if it can arise only from recombination with its own in a correlated fashion is under debate. For the parameters of Fig. 4, we find that the maximum excursion of an electron relative to the hole until their first recollision is between 15 and 27 lattice cells, depending on field strength, which is determined by the time of birth. For example, in the case of 27 cells, the electron moves a maximum of 14 lattice cells, whereas the hole moves 13 lattice cells in the opposite direction. For the short trajectories, the maximum excursion ranges between 14 (cutoff) and 4 lattice cells depending on birth time and harmonic order. The ionization rate is around $10^{-3}$ corresponding to an average distance of 10 atomic cells between neighboring excitonic electron-hole pairs. As a result, neighboring excitons can encounter each other during the first recollision. However, even when neighboring excitons collide, results from atomic HHG [29] indicate that this will not lead to coherent harmonic emission. Further work will be required to establish whether solids behave similar to atomic gases in this respect.

Finally, the coefficient $\delta$ in Table II is approximately independent of electric field strength, up to the field strength for which the cut-off approaches the maximum band gap (see Table II). However, we find that the birth and recollision times shift for increasing fields, a behavior that has not been observed for atomic HHG. The variation of the trajectory with field strength arises from the cutoff electrons (and holes) exploring the nonparabolic part of the band structure. This feature will be further explored in the next section.

\section{TRAJECTORY ANALYSIS}

The classical trajectory evolution is analysed in the following for the model $\mathrm{ZnO}$ crystal with $\mathrm{NN}$ bands. The trajectories are propagated in a CW laser field with frequency $\omega=0.014$. The effect of the pulse envelope on the trajectory can be safely neglected.
Figures 5(a)-5(d) show the harmonic photon energy as a function of the recollision time for increasing field strengths. Electrons born at a specific time can recollide with the hole multiple times. These higher returns are color coded from the first (blue) to the fifth (black). The thin green line represents the energy of the first recollision in an atomic system, for a field strength that yields the same cutoff; for the atomic system it is assumed that the ionization potential $I_{p}=E_{g}$.

Whereas the atomic trajectories are independent of the field strength, in a crystal they are not. For example, the blue line in Fig. 5(b), calculated with $F_{0}=0.0053$ a.u., is distorted with respect to that of Fig. 5(a), where $F_{0}=0.0046$ a.u. For the higher field, the cutoff originates from trajectories that are born and recollide almost $10^{\circ}$ later in the cycle as compared to the lower field case. The differences between the solid and atomic case become even more pronounced as the field is further increased, see Figs. 5(c) and 5(d). This striking difference is a consequence of the nonparabolic band dispersion. For higher field strengths electron and hole start to explore the nonquadratic part of the band. When this happens the quadratic energy-momentum relation and therewith the velocity is strongly modified and the trajectories are no longer invariant with respect to $F$.

Similar to atomic HHG, the cutoff energy separates two sets of trajectories that recollide within one optical cycle and contribute to the same harmonic. Those that travel a longer time, the long trajectories, are born after the peak of the field but before the birth time of the cutoff trajectory $t_{c}^{\prime}$; the short trajectories instead are born at times later than $t_{c}^{\prime}$.

For field strengths $F_{0} \leqslant 0.0053$ a.u., the cutoff is below or equal to the maximum possible photon energy (black dasheddotted line), which is determined by the maximum energy difference between valence and conduction bands. Here the long and short branches are clearly identifiable. When the intensity is increased beyond this point, see Figs. 5(c) and 5(d), the cutoff saturates. As a result, the simple long/short trajectory picture is lost. For $F_{0}=0.0058$ a.u., Fig. 5(c), the first recollision is prohibited for part of the long trajectories and does not occur continuously throughout the cycle. During the part of the cycle where first returns are prohibited, higher re-encounters occur.

For $F_{0}=0.008$ a.u., Fig. 5(d), the long trajectory does not recollide at all, but short trajectories can recollide a second time within the optical cycle. This field strength corresponds to $0.43 \mathrm{~V} / \AA$ in the crystal. With the refractive index $n=1.8992$ of $\mathrm{ZnO}$ at $\lambda=3.62 \mu \mathrm{m}$, it corresponds to $F_{\mathrm{v}}=0.62 \mathrm{~V} / \AA$, which has been reached in experiments [4].

The classical trajectories are validated by comparison with the simulated interband harmonic spectrum. Figures $5(\mathrm{e})-5(\mathrm{~h})$ show the spectral intensity (color coded) as a function of time (horizontal axis) and harmonic order (vertical axis). A windowed Fourier transform, identical to that used in Sec. $\mathrm{V}$, allows for the investigation of the harmonic emission with sub-cycle temporal resolution. For the simulations, the NN approximation is used. The dephasing time is $T_{2}=T_{0} / 2$ (5.4 fs). Classical trajectories recolliding within the optical cycle are represented by the solid white lines. Since trajectories are launched at each peak of the laser field, the simulation shows replicas of the emission delayed by multiples of a 


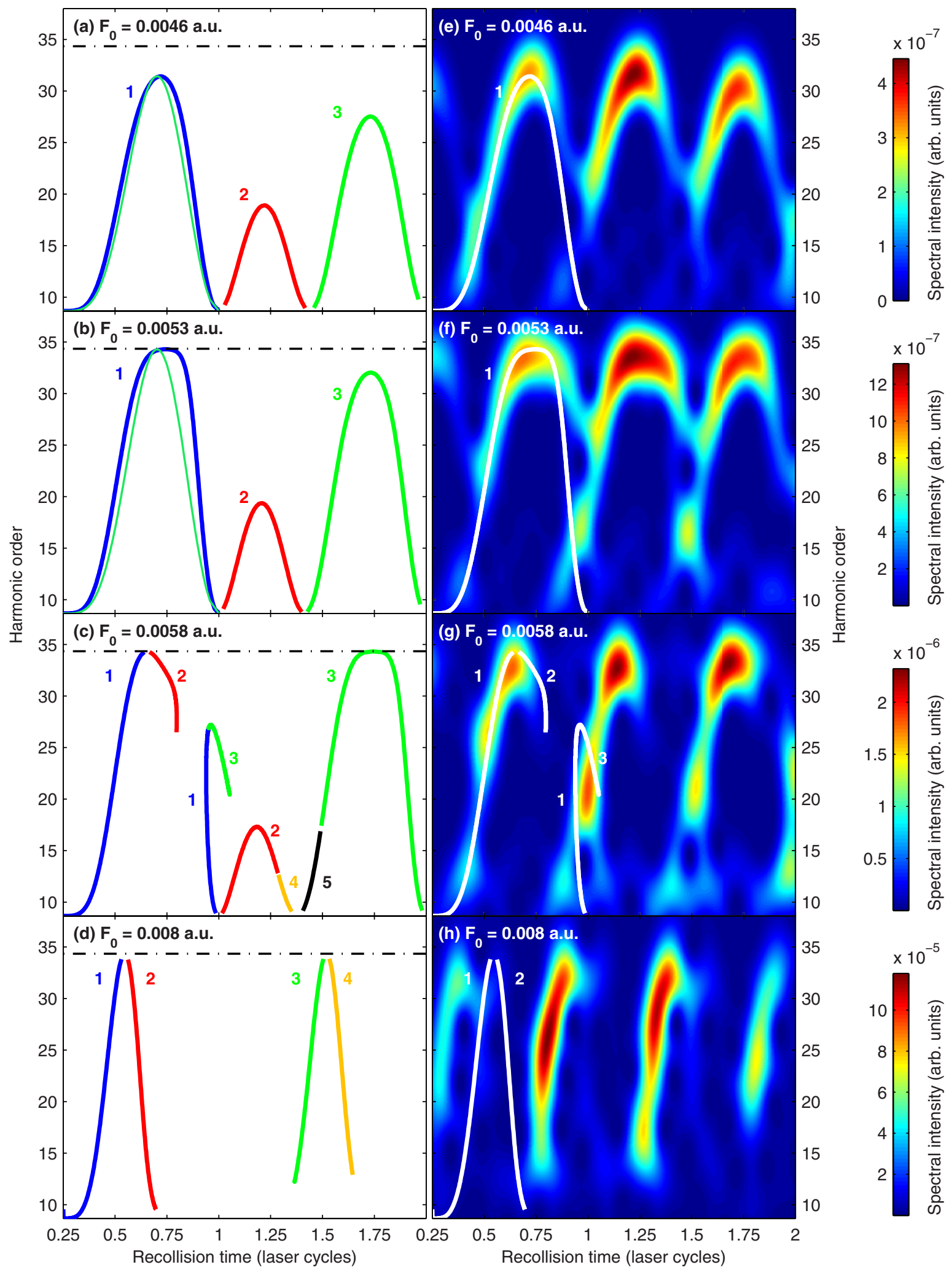

FIG. 5. (Color online) [(a)-(d), left panels] High harmonic photon energy as a function of recollision time for a ZnO crystal with NN bands for various field strengths. Subsequent recollision events are numbered sequentially and color coded as follows: first, second, third, fourth, fifth. The thin green line is the recollision energy of atomic HHG normalized to the cutoff of the crystal case. The dashed-dotted black line is the maximum band gap at the edge of the Brillouin zone. [(e)-(h), right panels] Simulated high harmonic spectral intensity (color code) as a function of harmonic order (vertical axis) and time (horizontal axis). The classical trajectories recolliding within the cycle, found in (a)-(d), are the solid white lines and they are numbered like in (a)-(d). Replicas of this emission at following laser half-cycles arise from the intrinsic periodicity of the HHG process. 


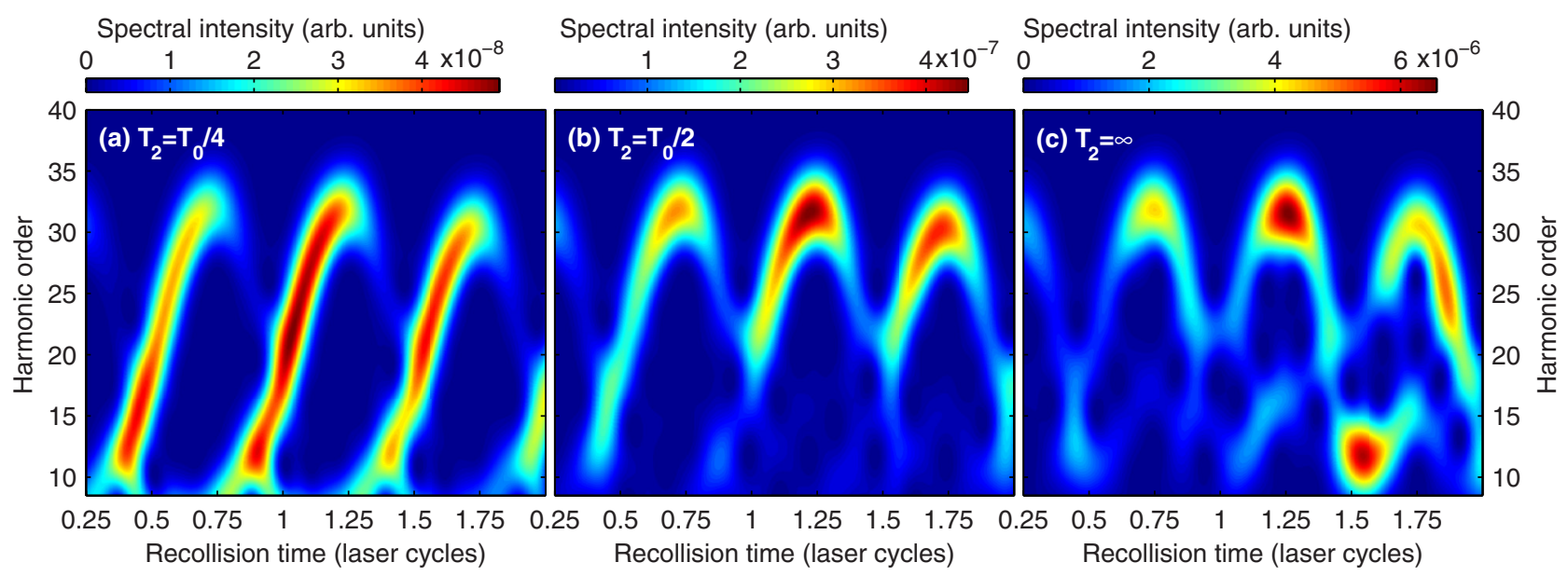

FIG. 6. (Color online) Harmonic order vs time for three different dephasing times: (a) $T_{2}=T_{0} / 4$, (b) $T_{0} / 2$, and (c) $\infty$. The laser frequency is $\omega=0.014$ a.u. and the field strength is $F_{0}=0.0046$ a.u. The shorter the dephasing time the weaker the long trajectories are. In (c), multiple returns significantly modify the recollision.

laser half-cycle. These replicas are not plotted in the trajectory analysis curves.

Emission of high harmonic photons happens four times per cycle, corresponding to a set of one long and one short trajectories for each half cycle. Seven recollision events are thus observed in the 1.75 cycles window considered in Fig. 5. The long and short branches come out clearly up to $F_{0}=0.0053$ a.u. The small discrepancies, such as the higher cutoff photon energy reached in the simulation, arise from approximations, such as the neglect of the ionization process in the classical trajectory calculation, see the previous section. Further, in Figs. 5(e)-5(f), we find a temporal overlap between long trajectories in one half-cycle and short trajectories of the next half-cycle. This results in spectral interference and modulation of the spectral intensity of the stronger short trajectories.

Figure 5(g) confirms the existence of the second recollision of the short branch within one half cycle that is predicted for $F_{0}=0.0058$ by the trajectory analysis. Furthermore, in agreement with trajectory analysis, the long branch is absent for harmonics above order 27.
For $F_{0}=0.008$ a.u., the differences between trajectory and numerical analysis are striking. Although the slope of the harmonic emission in time seems to agree with the short branch, all other trajectories vanish and the emission is shifted in time. At such high field strengths the ionized fraction reaches $2.5 \%$ at the end of the pulse. Clearly the low-ionization/depletion Keldysh limit, on which our analysis is based, breaks down. It appears that the interband current cannot be analysed on the basis of Eq. (6) and an extended model needs to be developed for the high-field regime.

Figure 5 also reveals the lack of higher order recollisions after the first return. In atomic HHG, long returns are suppressed by quantum diffusion resulting in a small recombination cross section between free electrons and atomic ground state. In solids, the short dephasing times $(\approx 5 \mathrm{fs})$ are responsible for suppressing higher-order returns. This was found in Fig. 2 and is confirmed by the trajectory analysis in Fig. 6, for $T_{2}=T_{0} / 4$ (a), $T_{0} / 2$ (b) and $T_{\infty}$ (c). For increasing dephasing times the spectral intensity of the long trajectories becomes stronger. When the dephasing time is long enough to allow returns longer than a laser cycle (c), multiple recollisions

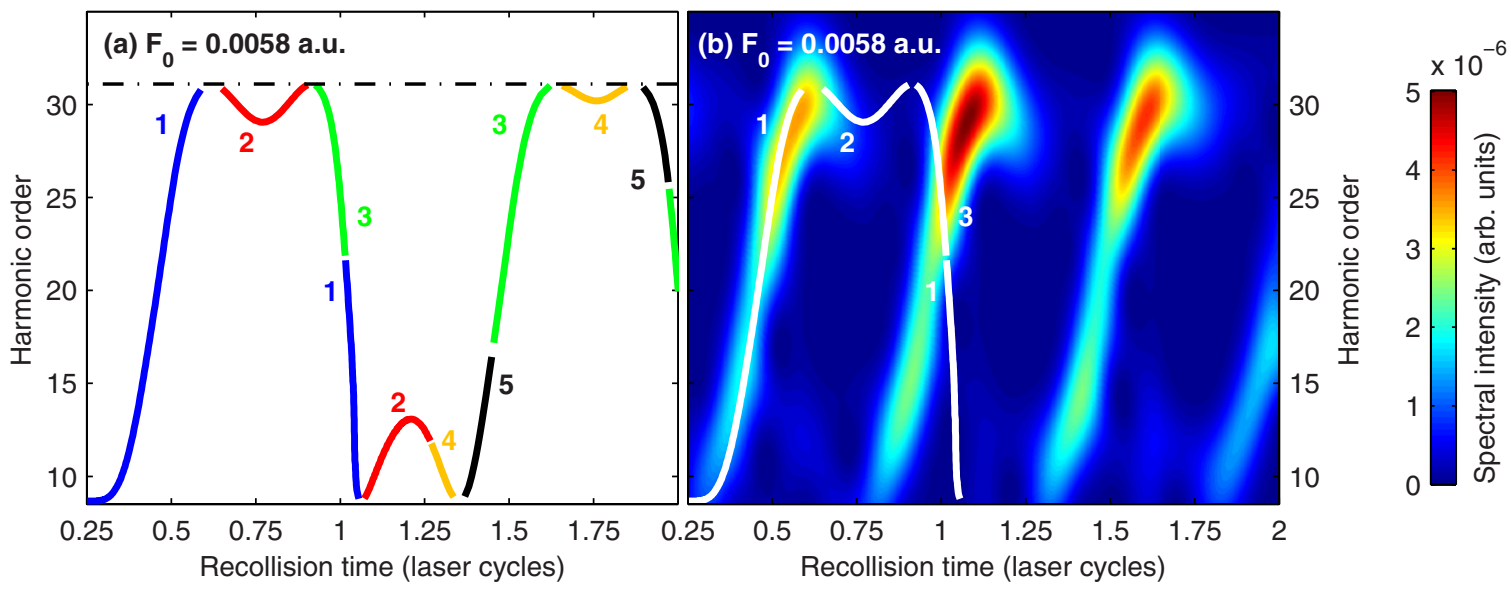

FIG. 7. (Color online) (a) and (b) Same plots as in Fig. 5, but for $\mathrm{ZnO}$ bands obtained from Ref. ([25]) at a field strength $F_{0}=0.0058$ a.u. The band parameters are reported in the caption of Fig. 2. 
interfere and lead to an aperiodic photon emission. This results in the loss of the clear harmonic structure in the spectrum. The simulation confirms the role of dephasing in suppressing multiple recollisions.

In Figs. 7(a) and 7(b), the importance of the band structure is investigated by comparing trajectory analysis results for NN approximated bands and for the full bands used in Sec. III. All other parameters are the same as in Figs. 5(c) and $5(\mathrm{~g})$ where the NN band has been used. Comparison between Figs. 7(a), 7(b) and 5(c), 5(g) reveals that HHG is quantitatively sensitive to the band structure for high field strengths, for which electron hole pairs explore a substantial fraction of the Brillouin zone. However, no qualitatively new features are introduced by using the full band structure.

\section{CONCLUSION}

We have compared the importance of the two main mechanisms contributing to HHG in solids over a wide range of wavelengths. For mid-IR lasers, generation is dominated by interband emission and it is driven by a three step process similar to atomic HHG. In contrast, for far-IR (THz) pump lasers, intraband emission arising from nonlinear band velocities and ionization dominates. The main part of our theoretical investigation has focused on a semiclassical understanding of the interband current. Our analysis reveals that the trajectory picture of atomic HHG is applicable to solids with some modifications.

In the atomic case, a plot of harmonic order normalized to the cutoff versus recollision and/or birth time normalized to the laser period is universally valid, independent of the laser field strength. This remains true in solids provided the electron hole pair only explores the quadratic region of the band gap. However, for field strengths strong enough that the pair is driven into the nonquadratic part of the band, the field strength independence is lost.

The implications have to be considered for developing attosecond solid state technology and devices based on the following atomic $\mathrm{HHG}$ processes. In atomic $\mathrm{HHG}$, single attosecond pulses are routinely isolated from this pulse train by gating, allowing only one recollision to occur. Similar methods can be applied to solids allowing the generation of isolated subfemtosecond pulses (or light transients [30]). Furthermore, techniques that rely on the perturbation of the $\mathrm{HHG}$ process to gather information about the phase of the harmonic radiation [31,32], the strong-field ionization step [21], or about the generating medium itself $[19,20]$ can be directly translated from atoms to solids.

The harmonic cutoff relation derived from trajectory analysis reveals a connection between band gap parameters and cutoff. By measuring the cutoff as a function of the laser field strength we will be able to determine the band gap of materials as a function of crystal momentum.

For many of the applications outlined above, the maximum achievable harmonic order is important. The maximum cutoff is limited by the maximum band gap between valence and conduction bands. One route to realizing higher cutoffs is the use of large band gap materials, such as dielectrics. Another route might be to use higher laser intensities for which higher conduction bands will be populated [28].

Long linear molecules can be viewed as 1D solids. In such systems both, the solid and atomic HHG, mechanisms could be of relevance: an electron can either propagate along the chain or become ionized and propagate in vacuum, before recolliding with the hole. If the HHG mechanism can be identified in molecular chains, either solid state or atomic methods would become available to probe these systems. This will be the first step to extending attosecond research to larger molecules and, possibly, liquids. Interesting questions regarding the $\mathrm{HHG}$ process are: what is its efficiency in confined systems? Are there surface effects at the ends of the chain? Is recollision still dominating harmonic emission?

Finally, the three step process of HHG in solids can be expanded and tailored by using materials doped with impurities. In this case, the electron/hole will make a transition from the negatively/positively charged impurity to the conduction/valence band, propagate in the band and recombine with the impurity upon return. This process resembles atomic HHG even more closely. As a result, translation of atomic HHG to impurity doped solids will result in a novel tool for the investigation of impurities in condensed matter. As an example, it could be possible to image the atomic orbital of the initial state of the impurity by extending the method of molecular tomography [19] developed for atomic HHG.

\section{ACKNOWLEDGMENT}

The authors would like to thanks Pawel Hawrylak for insightful discussions.
[1] R. W. Boyd, Nonlinear Optics (Academic Press, San Diego, 2003).

[2] P. B. Corkum, Phys. Rev. Lett. 71, 1994 (1993).

[3] P. B. Corkum and F. Krausz, Nat. Phys. 3, 381 (2007).

[4] S. Ghimire et al., Nat. Phys. 7, 138 (2011).

[5] B. Zaks, R. B. Liu, and M. S. Sherwin, Nature (London) 483, 580 (2012).

[6] O. Schubert et al., Nat. Photon. 8, 119 (2014).

[7] F. Brunel, JOSA B 7, 521 (1990).

[8] D. Golde, T. Meier, and S. W. Koch, Phys. Rev. B 77, 075330 (2008).
[9] G. Vampa, C. R. McDonald, G. Orlando, D. D. Klug, P. B. Corkum, and T. Brabec, Phys. Rev. Lett. 113, 073901 (2014).

[10] N. W. Ashcroft and N. D. Mermin Solid State Physics (Holt, Rinehart and Winston, New York, 1976).

[11] D. Golde, M. Kira, T. Meier and S. W. Koch, Phys. Status Solidi 248, 863 (2011).

[12] A. F. Kemper, B. Moritz, J. K. Freericks, and T. P. Deveraux, New. J. Phys. 15, 023003 (2013).

[13] K. A. Pronin, A. D. Bandrauk, and A. A. Ovchinnikov, Phys. Rev. B 50, 3473 (1994).

[14] P. G. Hawkins and M. Yu. Ivanov, Phys. Rev. A 87, 063842 (2013). 
[15] E. Goulielmakis et al., Nature (London) 466, 739 (2010).

[16] A. L. Cavalieri et al., Nature (London) 449, 1029 (2007).

[17] S. Neppl, R. Ernstorfer, E. M. Bothschafter, A. L. Cavalieri, D. Menzel, J. V. Barth, F. Krausz, R. Kienberger, and P. Feulner, Phys. Rev. Lett. 109, 087401 (2012).

[18] A. Föhlisch et al., Nature (London) 436, 373 (2005).

[19] J. Itatani et al., Nature (London) 432, 867 (2004).

[20] C. Vozzi, M. Negro, F. Calegari, G. Sansone, M. Nisoli, S. De Silvestri, and S. Stagira, Nat. Phys. 7, 822 (2011).

[21] D. Shafir et al., Nature (London) 485, 343 (2012).

[22] K. T. Kim, C. Zhang, T. Ruchon, J.-F. Hergott, T. Auguste, D. M. Villeneuve, P. B. Corkum, and F. Quéré, Nat. Photon. 7, 651 (2013).

[23] A. V. Mitrofanov, A. J. Verhoef, E. E. Serebryannikov, J. Lumeau, L. Glebov, A. M. Zheltikov, and A. Baltuška, Phys. Rev. Lett. 106, 147401 (2011).
[24] L. V. Keldysh, J. Exp. Theor. Phys. 47, 1945 (1964) [Sov. Phys. JETP 20, 1307 (1965)].

[25] M. Goano, F. Bertazzi, M. Penna, and E. Bellotti, J. App. Phys. 102, 083709 (2007).

[26] M. Lewenstein, P. Balcou, M. Y. Ivanov, A. L'Huillier, and P. B. Corkum, Phys. Rev. A 49, 2117 (1994).

[27] O. Smirnova and M. Ivanov, Attosecond and XUV Spectroscopy: Ultrafast Dynamics and Spectroscopy, edited by T. Schultz and M.Wrakking (Wiley-VCH, Weinheim, Germany, 2014), Chap. 7.

[28] P. G. Hawkins, M. Y. Ivanov, and V. S. Yakovlev, arXiv:1409.5707.

[29] H. Niikura, F. Légaré, R. Hasbani, M. Y. Ivanov, D. M. Villeneuve, and P. B. Corkum, Nature (London) 421, 826 (2003).

[30] E. Goulielmakis et al., Science 305, 1267 (2004).

[31] K. T. Kim et al., Nat. Phys. 9, 159 (2013).

[32] N. Dudovich et al., Nat. Phys. 2, 781 (2006). 